\title{
Effects of Concentrated Growth Factor on the Proliferation, Migration, and Osteogenic Differentiation of Rat Bone Marrow Mesenchymal Stem Cells: An in vitro study
}

\section{Xiang Yu}

The first Affiliated Hospital of Guangzhou University of Chinese Medicine

\section{Hui Ren}

The First Affiliated Hospital of Guangzhou University of Chinese Medicine

\section{Qi Shang}

Guangzhou University of Chinese Medicine

\section{Gengyang Shen}

The First Affiliated Hospital of Guangzhou University of Chinese Medicine

\section{Kai Tang}

Guangzhou University of Chinese Medicine

\section{Fuyong Yu}

Guangzhou University of Chinese Medicine

\section{Guifeng Chen}

Guangzhou University of Chinese Medicine

\section{Zhida Zhang}

Guangzhou University of Chinese Medicine

\section{Wenhua Zhao}

Guangzhou University of Chinese Medicine

\section{Peng Zhang}

Guangzhou University of Chinese Medicine

\section{De Liang}

The First Affiliated Hospital of Guangzhou University of Chinese Medicine

Xiaobing Jiang ( $\nabla$ spinedrjxb@sina.com )

Guangzhou University of Traditional Chinese Medicine First Affiliated Hospital https://orcid.org/00000002-8009-0282

\section{Research}


Keywords: Concentrated growth factor, bone marrow mesenchymal stem cells, BMP-2/Smad signaling pathway, bone tissue engineering

Posted Date: May 21st, 2020

DOI: https://doi.org/10.21203/rs.3.rs-29138/v1

License: (c) (i) This work is licensed under a Creative Commons Attribution 4.0 International License. Read Full License 


\section{Abstract \\ Background}

Concentrated growth factor (CGF) has been reported to be effective in bone formation or soft/hard tissue healing in recent years. Despite a few studies regarding the effects of CGF on the proliferation, migration, and osteogenic differentiation of BMSCs, their underlying mechanisms are not fully understood. The purpose of this study is to investigate the effects and possible mechanisms of CGF on the proliferation, migration, and osteogenic differentiation of rat-derived bone marrow mesenchymal stem cells (BMSCs) in vitro.

\section{Methods}

CGF was extracted from the Sprague Dawley (SD) rats by venipuncture of the abdominal aortic vein, and scanning electron microscopy (SEM) was used for the structural characterization. The release of bone morphogenetic protein 2 (BMP-2) from CGF was measured over the periods of $1 \sim 14$ days, using the enzyme-linked immunosorbent (Elisa) assay. Cell Counting Kit-8 (CCK-8) assay was used to measure cell proliferation. Migration capacity was analyzed using the transwell assay. The osteogenic differentiation and mineralization ability were determined by Alkaline phosphatase activity (ALP) staining and Alizarin Red staining respectively. Quantitative real-time PCR (RT-qPCR), was used to evaluate the mRNA expression levels of Runx2, Ocn, Smad1, and Smad5 after culture for 14 days. Further, the protein expression of BMP-2, phosphorylated-Smad1/5 (p-Smad1/5), and Smad1/5/8 was determined by Western blot after a 14-day cell culture.

\section{Results}

The SEM analysis showed a porous and dense three-dimensional fibrin network in CGF. The Elisa assay showed that BMP-2 was released from CGF extract for more than $14 \mathrm{~d}$, and it reached a peak at the time point of $5 \mathrm{~d}$. The cell densities of the CGF group at the different concentrations $(5 \%, 10 \%$, and $20 \%)$ were significantly higher than that of the control group at the periods of day 1 to day $5(p<0.05)$. Moreover, the number of migratory cells of the CGF group was greater than that of the control group at $24 \mathrm{~h}$. ALP activity analysis and Alizarin Red staining results demonstrated that CGF may successfully induce osteogenic differentiation of BMSCs. Moreover, the RT-qPCR results showed that CGF extracts dramatically enhanced the mRNA expression levels of Runx2, Ocn, Smad1, and Smad5 in BMSCs at days $14(p<0.05)$. Furthermore, Western blot results showed that CGF extracts markedly up-regulated the protein expression levels of BMP-2, p-Smad1/5, and Smad1/5/8.

\section{Conclusions}


CGF can promote the proliferation, migration, and promote the osteogenic differentiation potential of BMSCs in vitro. The BMP-2/Smad signaling pathway was involved in the osteogenic differentiation and mineralization of BMSCs induced by CGF. Therefore, CGF has good application potential in tissue engineering for bone regeneration and repair.

\section{Background}

Recently, concentrated platelets have been used in bone tissue and regenerative medicine fields because of their high growth factor contents. The commonly used materials include platelet-rich plasma (PRP), and platelet-rich fibrin (PRF), and concentrated growth factors (CGF) [1-6]. The PRP and PRF have been used alone or with graft materials to increase bone formation and to promote the bone defects healing $[2$, $7,8]$. The preparation process of PRP is complicated, as it requires several complex isolation procedures and activation agents, such as thrombin and calcium chloride. CGF is the third generation of platelet concentrate product, introduced by Sacco in 2006 [9]. It appears to have more abundant growth factors and a more rigid fibrin structure than PRP and PRF, due to the special centrifugation process $[10,11]$. A number of previous studies have demonstrated that CGF's potentials to promote bone formation, tissue repair and regeneration $[6,11-18]$.

BMSCs have played a key role in cell-based therapy and tissue engineering due to the multiple differentiation potential of them in resent years [19-21]. BMSCs are the preferred candidate cell resource for bone tissue engineering and cell-based therapies because they can differentiate into bone-forming osteoblasts and play a crucial role in bone regeneration [22]. Nevertheless, studies regarding the effects of CGF on the proliferation, migration, and osteogenic differentiation of BMSCs are scarce, and their underlying mechanisms have not yet been explored in depth. Thus, for the further application of CGF, it's of great value to clarify the effect and mechanism of CGF on the proliferation, migration, and osteogenic differentiation of BMSCs. Several studies have demonstrated that the BMP-2/Smad signaling pathway is involved in bone remodeling processes including osteoblast differentiation and osteoclastogenesis [23, 24]. Bone morphogenetic proteins (BMPs), as members of the TGF- $\beta$ family of signaling molecules, are capable of regulating a diverse range of biological processes and gene expressions, through their activation of Smad1, Smad5, or Smad8 proteins [23]. These Smad transcription factors achieve a layer of functional specificity in different cell types largely through actions with additional transcriptional regulatory molecules. In the BMP-2/Smad signaling, phosphorylated Smad1/5/8 assemble into complexes with Smad4 and then translocate into the nucleus to activate Runx2-mediated regulation of osteoblast differentiation [25]. Thus, BMP-2/Smad signaling pathway has close relationship with BMSCs osteogenic differentiation [26]. However, the effect of CGF extracts on BMP-2/Smad signaling pathway during osteogenic differentiation of BMSCs has not yet been explored.

In this study, we aim to investigate the effects and the possible mechanism of CGF on BMSCs proliferation, migration, osteogenic differentiation and mineralization. For the first time, we evaluated the expression of BMP-2/Smad signaling pathway related genes or proteins during the period of CGF promoting osteogenic differentiation of BMSCs. Our results showed that CGF can positively promote the 
proliferation, migration, osteogenic differentiation and mineralization of BMSCs in vitro. Moreover, the BMP-2/Smad pathway was involved in the osteogenic differentiation and mineralization of BMSCs induced by CGF. These results indicate that CGF has good application potential for biotechnological and clinical applications in the field of bone tissue engineering and regeneration medicine.

\section{Materials And Methods}

\section{Preparation of CGF}

This research was approved by the Clinical Research Ethics Committee of the First Affiliated Hospital of Guangzhou University of Chinese Medicine (Guangzhou, People's Republic of China). CGF was extracted from the healthy Sprague Dawley (SD) rats by venipuncture of the abdominal aortic vein. In brief, the blood was collected in sterile Vacuette tubes (Beijing Ruidexy Medical Technology Limited, China) without anticoagulant solutions. CGF was prepared according to Sacco's protocol [27]. These tubes were then immediately centrifuged (Medifuge CGF, MF200,Silfradent Srl, Italy) using a program with the following characteristics: This centrifuge device used a program with the characteristics: $2,700 \mathrm{rpm}$ $2 \mathrm{~min}, 2,400 \mathrm{rpm} 4 \mathrm{~min}, 2,700 \mathrm{rpm} 4 \mathrm{~min}$, and 3,000 rpm $3 \mathrm{~min}$ [28]. Immediately after CGF preparation, a CGF extract was prepared. Briefly, CGF was transferred into a syringe and was gently compressed in the syringe so a liquid fraction (CGF extract) can be fabricated, the extracts were filtered by a $0.22 \mu \mathrm{m}$ sterile syringe filter (Solarbio, China). The final CGF concentration (5\%, 10\% and $20 \%$ ) in the experimental groups was calculated on the basis of the volume of CGF that was added to the total volume of culture medium containing $10 \%$ fetal bovine serum (FBS). The control group only received $10 \%$ FBS.

\section{Enzyme-linked Immunosorbent Assay (ELISA)}

Growth factors extracted from the CGF were prepared according to previously reported protocols with slight modifications [29]. In brief, the CGF clot was soaked in $1 \mathrm{~mL}$ DMEM (Cyagen Biosciences Inc. Guangzhou, China) without FBS in a $15 \mathrm{~mL}$ flacon tube and incubated at $37^{\circ} \mathrm{C}$. The medium was collected continuously for 14 days. Following collection, $1 \mathrm{~mL}$ fresh DMEM was added to the tube. All the collected extracts were stored at $-80^{\circ} \mathrm{C}$. Finally, the Elisa kit (Elabscience Biotechnology Co., Ltd) was used to quantify the representative growth factor of BMP-2 released from the CGF extract according to the manufacturer's instructions. All the assays were performed in triplicate.

\section{The structure of CGF tested by Scanning electron microscope (SEM)}

To verify the structure of CGF, CGF was lyophilized and examined by SEM (fei-q25, American FEI company). In brief, CGF was pre-frozen at $-60^{\circ} \mathrm{C}$ for $24 \mathrm{~h}$ and then dehydrated for $24 \mathrm{~h}$ using a freeze dryer (SCIENTZ-10N, Ningbo, China). The samples were then observed, and representative images were captured. 


\section{Isolation, Culture And Identification Of Rat BMSCs}

BMSCs were harvested from 2-week-old healthy SD female rats. After euthanasia, bone marrow was flushed out from tibia and femur with SD Rat Bone Marrow Mesenchymal Stem Cell Basal Medium supplemented with 10\% (v/v) Fetal Bovine Serum, 1\% (v/v) Glutamine and 1\% (v/v) PenicillinStreptomycin (Cyagen Biosciences Inc. Guangzhou, China) in standard sterile condition. Cells were incubated under the condition of $5 \% \mathrm{CO} 2$ at $37^{\circ} \mathrm{C}$ in a cell incubator. Cells were passaged every 3 days using $0.25 \%(w / v)$ trypsin-EDTA solution. The third passage cells were used to perform the flow cytometry identification according to the method as reported [30]. After identification, the third passage cells were used in our experiments.

\section{Cell Proliferation Assay}

To detect the capacity of CGF promoting cell proliferation, CCK-8 assay was performed. In brief, BMSCs were seeded in 96 -well plates at a density of 2000 cells/well in the culture medium. After $24 \mathrm{~h}$ of culture, DMEM medium was replaced with different concentrations $(5 \%, 10 \%$, and $20 \%)$ of CGF extracts or FBS for 1,3 , and 5 days in the same conditioning culture $\left(37^{\circ} \mathrm{C}, 5 \% \mathrm{CO} 2\right)$. The cell proliferation rates were determined with CCK-8 (Solarbio, China) using a microplate reader (Bio-Rad, Hercules, USA) at a wavelength of $450 \mathrm{~nm}$. Eight repetitive wells were used for each group, the maximum and minimum values were excluded, and the remaining data were used for statistical analysis.

\section{Migration Assay}

To detect the capacity of CGF on the migration of BMSCs, the transwell migration assay was performed. The transwell migration assay was performed according to the method reported [31]. In brief, BMSCs were suspended at a density of $1 \times 10^{4} /$ well and loaded into the top chamber of a 24-well, $8 \mu \mathrm{m}$ pore-size transwell plate (Corning, NY, USA). Then, complete medium from different groups with CGF or medium was added to the lower chamber. After $24 \mathrm{~h}$, un-migrated cells that remained in the upper chambers were removed by wiping the top of the insert membranes with cotton swabs, while the migrated cells that passed through the membrane pores were stained with $0.5 \%$ crystal violet for several minutes and counted under an optical microscope (BX53, OLYMPUS CORPORATION, Japan).

\section{Alizarin Red Staining And Alkaline Phosphatase Activity Assay}

To test the osteogenic differentiation and mineralization ability of CGF on BMSCs, Alkaline phosphatase activity assay and Alizarin Red Staining were performed after osteogenic differentiation for 14 days. According to the CCK-8 and migration results, 10\% CGF extract was selected to perform the osteogenic differentiation experiment. Briefly, BMSCs were seeded in 12-well plates at a density of $2 \times 10^{4} \mathrm{cell} / \mathrm{s} / \mathrm{cm}^{2}$. 
After incubation for $12 \mathrm{~h}$ in complete medium, the medium was changed to medium containing with $0 \%$, $5 \%, 10 \%$, or $20 \%$ CGF extract, the cells were divided into 4 groups: as follows: i) The control group (Con), cultured with the complete medium without osteogenic induction medium; ii) 10 CGF\% group, cultured with the medium supplemented with $10 \%$ CGF extract without osteogenic induction medium; iii) Osteogenic induction group (OI), cultured in the presence of osteogenic induction medium; and iv) $\mathrm{OI}+$ 10\% CGF group, cultured with the medium supplemented with 10\% CGF extract in the presence of osteogenic induction medium. After cultured for 14 days, Alizarin Red staining and ALP activity were performed. For Alizarin Red staining, after gently rinsing with $\mathrm{ddH}_{2} \mathrm{O}$, the cells were stained in a solution of $2 \%$ Alizarin Red at pH 4.1 for 20 min and then washed with $\mathrm{ddH}_{2} \mathrm{O}$. The samples were air-dried, and images were captured under a light microscope (BX53, OLYMPUS CORPORATION, Japan). Additionally, the bound alizarin red was dissolved in 200 II $100 \mathrm{mM}$ hexadecylpyridinium chloride (Cyagen Biosciences Inc. Guangzhou, China) and the absorbance (OD value) of the supernatant was measured at $578 \mathrm{~nm}$. After staining, plates were digitally photographed and the acquired images were analyzed.

For the ALP activity assay, the cell supernatant in each group was collected after cultured for 14 days. ALP activity of BMSCs was measured using Lab Assay ALP (Cyagen Biosciences Inc. Guangzhou, China), according to the manufacturer's protocol. ALP activity was normalized to total protein determined by BCA protein assay kit (Beyotime). The optical density (OD) of the ALP protein was measured at $520 \mathrm{~nm}$ according to the manufacturer's instruction. ALP activity was calculated using the ALP level normalized to the total protein.

\section{Quantitative Real-time Pcr (RT-qPCR)}

The mRNA expressions of Runx2, Ocn, Smad1, and Smad5 were detected by RT-qPCR. Briefly, BMSCs were cultured with cell culture media without CGF extract, or with 10\% CGF extract, OI, and OI +10\% CGF extract. After 14 days of culture, the cells were collected to perform TR-qPCR and Western Blot. For the RTqPCR, total RNA was isolated from cells by use of the RNeasy mini Kit (Qiagen, Valencia, CA, USA), in accordance with the manufacturer's instructions. CDNA was synthesized by use of the SuperScript III First-Strand synthesis system (Invitrogen). This CDNA was then analyzed by real-time PCR analysis by use of the 7900HT fast real-time PCR system (Applied Biosystems, Tokyo, Japan). The SYBR green assay with SYBR Premix EX taq (Tli RnaseH plus; TaKaRa Bio, Otsu, Japan) was used for amplification of all target transcripts. Expression values were normalized to $\beta$-actin. The sequences of the specific primers for the target genes were listed as follows (Sangon Biotech, China): Runx2 (forward: 5'GACCAGTCTTACCCCTCCTA-3' and reverse: GGCAGTGTCATCATCTGAAA-3'); Ocn (forward: 5'ATGGCGTATTAGAGGCAGCA-3' and reverse: 5'-GGTTTCGTGAAGAGCGCCA-3'); Smad1 (forward: 5'CAGCGTGTTGGTGGATGGT - 3' and reverse: 5'-CCGTGGTGGGATGAAAGC - 3'); Smad5 (forward: 5'AACACCAGGCGGCACATCGG - 3' and reverse: 5'-GACGGTGGTGGGGTGGAAGC-3'); $\beta$-actin (forward: 5'GGAGATTACTGCCCTGGCTCCTA - 3' and reverse: 5'-GACTCATCGTACTCCTGCTTGCTG - 3'). 


\section{Western Blot}

The protein expressions of BMP-2, p-Smad1/5/8, and Smad1/5/8 were detected by western blot. Briefly, the cell samples were lysed in RIPA lysisbuffer (Beyotime, China). Protein concentrations were measured using a BCA protein kit (Beyotime, China). Twenty microliters of protein were loaded per lane on a $10 \%$ SDS-PAGE gel for electrophoresis and then transferred to $0.22 \mathrm{~mm}$ PVDF membranes (Bio-Rad, USA).

\section{Statistical analysis}

All analyses were performed using SPSS 22.0 software (IBM Corp., Armonk, NY, USA). All experiments were performed at least in three independent repeats. All data are shown as the mean $\pm S D$, a two-sided unpaired Student's t-test or analysis of variance (ANOVA) followed by Tukey's test for multiple comparisons were used for statistical analysis of differences between groups. A statistical difference between experimental groups was regarded as significant when the $p$ value was $<0.05$.

\section{Results}

\section{CGF fabrication and its structure}

Blood from auxiliary veins of SD rats were extracted and centrifuged using the Silfradent system (Fig. 1a). The blood in the tube after centrifugation (Fig. 1b), three layers were observed at the end of the process, the CGF clot was acquired from the middle layer (white arrow). CGF gel was separated (Fig. 1C, d). SEM showed the structure of CGF, a lot of fibrous proteins were shown in a low magnification (Fig. 1e). Moreover, the freeze-drying CGF showed porous structure in a high magnification (Fig. 1f).

\section{The release profile of BMP-2 from CGF}

The secretion of BMP-2 from CGF was measured by ELISA in this study. A sustained-release test showed that CGF continuously released BMP-2 for over 14 days (Fig. 2). The level of BMP-2 increased from $99.333 \mathrm{pg} / \mathrm{ml}$ on Day 1 to $161.974 \mathrm{pg} / \mathrm{ml}$ on Day 5 . Subsequently, the level slowly decreased to 14.667 $\mathrm{pg} / \mathrm{ml}$ at the time point of day 14 .

\section{The Successful Identification Of BMSCs}

P0 passage BMSCs showed short bar shapes, and P1, P3 passage of BMSCs adopted a uniformly spindle-shaped population (Fig. 3a). Flow cytometry revealed that BMSCs expressed the surface markers CD29 and CD90 with the positive percentage of $95.6 \%$ and $97.6 \%$ respectively, but not CD45 and CD34 (with the negative percentage of $1.9 \%$ and $2.1 \%$ ) (Fig. 3b). 


\section{CGF Promotes BMSCs Proliferation}

As the CCK-8 results showed, cell proliferation was promoted, in a concentration-dependent fashion, by treatment with a CGF extract compared with the effect of serum addition. The effect of different concentration of CGF extract on cell proliferation showed a higher trend from day 1 to day 3 , and a significant difference between the effect of CGF and serum on cell proliferation was observed at all concentrations (5\%, 10\%, and 20\%) ( $p \forall 0.05, p \otimes 0.01, p \otimes 0.001)$ at all-time points (Fig. 4), while, the effect of a $20 \%$ CGF extract was inferior to those of the $10 \%$ group at every time point, and there were significant differences between low concentration (5\% CGF, 10\% CGF) and high concentration (20\% CGF) at the time point of day 3 and day $5(p \otimes 0.05, p \otimes 0.01)$.

\section{CGF Promotes BMSCs Migration}

The results of transwell assay revealed that the different concentrations $(5 \%, 10 \%$, and $20 \%)$ of CGF promoted the BMSCs migration, and 10\% CGF showed the most obvious promotion effect on cell migration (Fig. 5a). Meanwhile, the migratory cell numbers in each concentration of CGF groups were significantly higher than that of the control groups ( $0 \%$ CGF) after $24 \mathrm{~h}$ incubation $(p<0.001)$ (Fig. $5 \mathrm{~b})$. However, the migratory cell numbers in $20 \%$ CGF group was markedly decrease than that of $5 \%$ and $10 \%$ CGF group $(p<0.001)$.

BMP-2/Smad signaling pathway was involved in the osteogenic differentiation and mineralization induced by CGF

Alizarin Red staining and ALP activity revealed that the mineralization area and the ALP activity in the experimental groups (including 10\% CGF, OI, and OI + 10\% CGF groups) were significantly larger or higher compared to the control groups (without CGF extract) after incubation for 14 days $(p<0.05)$ (Fig. $6 a-c)$. Moreover, the RT-qPCR results showed that the experimental groups (including 10\% CGF, OI, and OI + 10\% CGF groups) significantly up-regulated the mRNA expression of Runx2, Ocn, Smad1, and Smad5 ( $p$ \& 0.001) (Fig. 6d-g). Besides, the Western blot results showed that the protein expressions of BMP-2, pSmad1/5/8, Smad1/5/8 were apparently enhanced in the experimental groups (10\% CGF, OI, and OI + $10 \%$ CGF groups) compared to the Con group (Fig. 6h). Meanwhile, the expression ratios of p-Smad1/5/8 : Smad1/5/8 and BMP-2: $\beta$-actin were significantly higher in the experimental groups than that of the

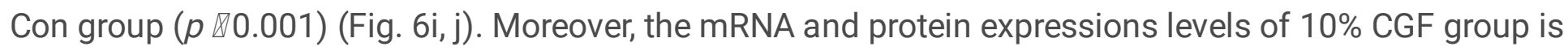
similar to OI group ( $p \otimes 0.05$ ). However, the OI + 10\% CGF group showed markedly higher mRNA (Runx2, Ocn, Smad1, and Smad5) and protein expressions (BMP-2, p-Smad1/5/8, Smad1/5/8; the ratio of p-

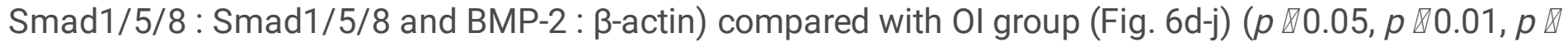
$0.001)$.

CGF promote osteogenic differentiation and mineralization of BMSCs partly via regulating BMP-2/Smad signaling pathway 
The possible underlying mechanism of CGF promote osteogenic differentiation and mineralization of BMSCs was summarized in Fig. 7. CGF secreted growth factors, such as BMP-2, then BMP-2 activated Smad1, Smad5, or Smad8 that, in turn, regulate gene expression. In the BMP-2/Smad signaling, phosphorylated Smad1/5/8 assemble into complexes with Smad4 and then translocate into the nucleus to activate Runx2-mediated regulation of osteoblast differentiation and mineralization.

\section{Discussion}

This study demonstrated that CGF extract effectively promoted the proliferation, migration, osteogenic differentiation potential of BMSCs. For the first time, we specified the mechanisms that CGF can induce BMP-2/Smad pathway during the osteogenic differentiation process.

CGF is the latest generation of platelet concentrate products, which is produced by centrifuging blood samples with a special centrifuge device. Due to the advanced extraction technology and specialized equipment (Medifuge, Silfradent Srl, Italy), the preparation process is simple and does not require any synthetic or catalytic substances. In the present study, the SEM results showed that CGF has a complex porous three-dimensional fibrin structure, which is consistent with others' results [14, 32, 33]. This dense fibrin network can provide a matrix for cell adherence and migration [33, 34]. BMSCs were isolated from the femurs and tibias of $2 \sim 3$-week-old SD rats, the properties of BMSCs were characterized using cell surface markers in this article. The flow cytometry identification results proved the cells that we cultured were BMSCs, evidenced by negative for CD45 and CD34 but positive for mesenchymal associated markers such as CD29 and CD90. Our results further revealed that with the concentrations of CGF from $5-10 \%$, significant promotion effects on the proliferation and migration of BMSCs in a dose-dependent manner were shown. However, higher concentrations of CGF extract do not guarantee stronger promoting effects, even will have inhibitory effects on the cultured cells $[19,35,36]$. In this article, the effect of a $20 \%$ CGF extract was inferior to those of the $10 \%$ group, which is consistent with other's results $[19,37,38]$. The reasonable explanations for this phenomenon might be two aspects. First, CGF extracts contains not only growth factors that favor osteogenic but also unosteogenic cytokines [39]. At high concentrations, the inhibitory effects of the inflammatory cytokines in CGF might play a dominant role [19]. Second, it has previously been proposed that $\mathrm{pH}$ value is affected by the change in platelet count, which may negatively affect cell proliferation [40]. So, the inability of high concentration of CGF to promote BMSCs proliferation may be associated with the $\mathrm{pH}$ value in the cell environment. While, the mechanism for the inhibitory effect of high concentrations of CGF are not completely clear, as CGF state may affect the capacity of CGF under different conditions, which needs to be further studied.

Moreover, our results also demonstrated that CGF could dramatically enhanced the osteogenic differentiation of BMSCs, which were evidenced by the ALP activity and Alizarin Red staining results. ALP is reported as a marker of osteoblast differentiation [41-43]. As the induction time increases and the osteogenic differentiation of cells progresses, its activity gradually increases [19]. The osteogenic activity of BMP-2 can be detected by measuring ALP activity $[44,45]$. Therefore, ALP activity was detected to explore the osteogenic function of the BMSCs induced by CGF can be evaluated. The results indicated 
that the ALP activity of the experimental group (10\% CGF, OI, and OI + 10\% CGF) were significantly higher than the control group (without CGF) after cultured for 14 days, suggesting that CGF can promote the osteogenesis of BMSCs.

Calcium salt levels change is not only a marker of bone cell proliferation and differentiation, but also a signal of the osteogenic potential of bone tissue. Therefore, the ability of osteoblasts to differentiate may be identified by determining the calcium salt deposition in the cell matrix of each group by Alizarin Red staining. Our results showed that, the number and density of the mineralized nodules were markedly higher in the experimental groups (10\% CGF, OI, OI + 10\% CGF) compared with the control group (without CGF and OI). These results further verified that CGF can promote the osteoblastic differentiation of BMSCs.

Understanding the underlying mechanisms are important for the development of novel therapeutic strategies and tissue engineering techniques. The BMPs family of proteins, through a cascade of downstream signals, regulate many aspects of skeletal development, including osteoblast and bone formation [46]. Gene responses to BMPs are mediated by Smad transcription factors after being phosphorylated by the BMP receptor complex $[47,48]$. BMP-2 promotes osteogenesis by binding to BMP2R and activating downstream osteogenic genes through a SMAD complex. Upon binding of BMP-2, the type-II receptor (BMPR-II) transphosphorylates the type-I receptor (BMPR-I) and the phosphorylated BMPR-I, in turn, phosphorylates a group of intracellular substrate signaling proteins collectively known as receptor-regulated Smads (Smad1, Smad5, and Smad8) [47, 49]. Next, phosphorylated Smad1/5/8 dissociates from BMPR-I, forms a heteromeric complex with a common-partner Smad (Smad4), then translocates from the cytoplasm into the nucleus where they regulate transcription of various target genes, including Runx2, to increase various kinds of osteogenic differentiation factors [46, 50]. Considering that BMP-2 is closely associated with the Smad pathway, and CGF can secret BMP-2, we hypothesized that the function of CGF on the osteogenic differentiation of BMSCs is possibly mediated by BMP-2/Smad signaling pathway. In order to investigate whether the BMP-2/Smad signaling pathway was involved in the induction of osteogenic differentiation of BMSCs by CGF, RT-qPCR and western blot analyses were conducted to quantify mRNA and protein expression of the experimental groups (10\% CGF, $\mathrm{OI}+10 \% \mathrm{CGF}$ ), pure mineralization group (OI) and control group (without CGF and OI) after osteogenic induction for 14 days. The RT-qPCR results indicated that expressions of Runx2, Ocn, Smad1, and Smad5 were significantly upregulated compared with the control group. The western blot results showed that the expressions of BMP-2, p-Smad1/5/8, and Smad1/5/8 were more apparent in the experimental groups compared to the control group after the intervention of CGF for 14days. Moreover, the significantly higher ratios of $p$-Smad1:Smad1/5/8 and BMP-2: $\beta$-actin were also shown in the experimental groups compared to the control group. These findings indicate that CGF likely promotes osteoblastic differentiation of GMSCs cells by regulating the BMP-2/Smad signaling pathway.

To the best of our knowledge, for the first time, we explored the effect of CGF on the BMP-2/Smad signaling pathway in promoting the osteogenic differentiation of BMSCs. The results will provide experimental basis and reference for elucidating the mechanism of CGF promoting osteogenic 
differentiation. However, some limitations are hardly involved in this article. First, we just detected the expression level of BMP-2 from CGF, the mechanism of osteogenic differentiation effect may be related with other growth factors secreted from CGF, which need to be further studied. Second, we preliminarily explored the function on the migration capability of BMSCs using CGF, nevertheless, the mechanism of the promoting migration capability wasn't clarified deeply in the present study, which need to be further clarified. Third, although the results in our article can initially demonstrated our hypothesis, the signal pathway inhibitors or promotors are also needed to further verify our hypothesis. In the future study, the signal pathway inhibitors or promotors of BMP-2/Smad signaling pathway, and the cell silencing or overexpression skills are expected to be applied to further identify the depth mechanism of CGF on the osteogenic differentiation of BMSCs in vitro or in vivo. Moreover, further studies are required to ascertain the effect and underlying mechanism on the proliferation, migration, and osteogenic differentiation induced by other growth factors from CGF. Furthermore, the therapeutic effect and mechanism of CGF on the diseases with bone defect (such as bone defect caused by long bone defects or osteoporotic fractures) needs further investigating in vivo studies.

\section{Conclusion}

In summary, the findings of the present study revealed CGF promoted the proliferation, osteogenic maturation, and mineralization of mesenchymal stem cells in a dose-dependent manner in vitro. Therefore, our investigation provided the in vitro experimental evidences of CGF as a promising material for certain applications in tissue engineering for bone regeneration and repair. However, the mechanisms underlying the regulation of the BMP-2/Smad signaling pathway by CGF remain to be fully elucidated.

\section{Abbreviations}

CGF®Concentrated growth factor冈BMSCs: Bone marrow mesenchymal stem cells; SEM: scanning electron microscopy; CCK-8: Cell Counting Kit-8; BMP-2: Bone morphogenetic protein 2; Elisa: Enzyme linked immunosorbent; ALP: Alkaline phosphatase activity; RT-qPCR: Quantitative real-time PCR; PRP: Plateletrich plasma; PRF: Platelet-rich fibrin; BMPs: Bone morphogenetic proteins; SD: Sprague Dawley; FBS: Fetal bovine serum; HADSC: Human adipose-derived stem cell; HUVECs: Human umbilical vein endothelial cells (HUVECs); GMSCs: Gingiva-derived mesenchymal stem cells.

\section{Declarations}

\section{Acknowledgements}

We thank Miss Fan Yang and Beijing Ruidexy Medical Technology Limited for providing Silfradent CGF system and related facilities and direction. We thank Changlu $X u$ and Dongwei Sun who are from University of California Riverside provided the English grammar revision. We also thank Lingnan Medical Research Center of Guangzhou University of Chinese Medicine for providing associated facilities. 


\section{Funding}

The project was generously supported by the grants from the project supported by Guangdong Province Universities and Colleges Pearl River Scholar Funded Scheme (GDUPS 2018), National Natural Science Foundation of China (81674000), Major discipline research project of Guangzhou University of Chinese Medicine in 2019, The youth scientific research training project of GZUCM (2019QNPY04), Excellent Young Scholars Project of China Association of Traditional Chinese Medicine (CACM-2017-QNRC1-01), Key Project of Basic Research and Applied Basic Research of Department of Education of Guangdong Province (2018KZDXM021), Special Research Project for the Construction of the National TCM Clinical Research Base of the State Administration of Traditional Chinese Medicine (JDZX2015078), Science and Technology New Star of Guangzhou Pearl river (201710010078), Excellent Doctoral Dissertation Incubation Grant of First Clinical School of Guangzhou University of Chinese Medicine (YB201702), Excellent Doctoral Dissertation Incubation Grant Guangzhou University of Chinese Medicine (A1-2606-19429-012), International Program for Postgraduates, Guangzhou University of Chinese Medicine (GJ201816), Excellent Young Scholars Project of the First Affiliated Hospital of Guangzhou University of Chinese Medicine (2017QN08, 2017TD08).

\section{Availability of data and materials}

Te data during the current study are available from the corresponding author on reasonable request.

\section{Authors' Contributions}

This study was based on the whole work of each author. All authors approved the final version of the manuscript. Xiang Yu, Hui Ren, and Qi Shang contributed equally to this work. All authors listed in the current study carried out the experiments, participated in the design of the study and performed the statistical analysis, conceived of the study, and helped to draft the manuscript. All authors read and approved the submitted manuscript.

\section{Ethics approval and consent to participate}

This research was approved by the the Clinical Research Ethics Committee of the First Affiliated Hospital of Guangzhou University of Chinese Medicine (Guangzhou, People's Republic of China).

\section{Consent for publication}

Not applicable.

\section{Competing interests}

The authors declare that they have no competing interests.

\section{Author details}


${ }^{1}$ Department of Spinal Surgery, The First Affiliated Hospital of Guangzhou University of Chinese Medicine, Guangzhou 510405, China. ${ }^{2}$ Guangzhou University of Chinese Medicine, Guangzhou, Guangzhou 510405 , China. ${ }^{3}$ Lingnan Medical Research Center of Guangzhou Univercity of Chinese Medicine, Guangzhou 510405, China.

\section{References}

1. Acar AH, Yolcu U, Gul M, Keles A, Erdem NF, Altundag KS. Micro-computed tomography and histomorphometric analysis of the effects of platelet-rich fibrin on bone regeneration in the rabbit calvarium. Arch Oral Biol. 2015;60:606-14.

2. Pripatnanont $P$, Nuntanaranont $T$, Vongvatcharanon $S$, Phurisat $K$. The primacy of platelet-rich fibrin on bone regeneration of various grafts in rabbit's calvarial defects. J Craniomaxillofac Surg. 2013;41:e191-200.

3. Dohan DM, Choukroun J, Diss A, Dohan SL, Dohan AJ, Mouhyi J, et al. Platelet-rich fibrin (PRF): a second-generation platelet concentrate. Part I: technological concepts and evolution. Oral Surg Oral Med Oral Pathol Oral Radiol Endod. 2006;101:e37-44.

4. Dohan DM, Choukroun J, Diss A, Dohan SL, Dohan AJ, Mouhyi J, et al. Platelet-rich fibrin (PRF): a second-generation platelet concentrate. Part II: platelet-related biologic features. Oral Surg Oral Med Oral Pathol Oral Radiol Endod. 2006;101:e45-50.

5. Dohan DM, Choukroun J, Diss A, Dohan SL, Dohan AJ, Mouhyi J, et al. Platelet-rich fibrin (PRF): a second-generation platelet concentrate. Part III: leucocyte activation: a new feature for platelet concentrates? Oral Surg Oral Med Oral Pathol Oral Radiol Endod. 2006;101:e51-5.

6. Kim TH, Kim SH, Sandor GK, Kim YD. Comparison of platelet-rich plasma (PRP), platelet-rich fibrin (PRF), and concentrated growth factor (CGF) in rabbit-skull defect healing. Arch Oral Biol. 2014;59:550-8.

7. Andia I, Maffulli N. Platelet-rich plasma for managing pain and inflammation in osteoarthritis. Nat Rev Rheumatol. 2013;9:721-30.

8. Alsousou J, Thompson M, Harrison P, Willett K, Franklin S. Effect of platelet-rich plasma on healing tissues in acute ruptured Achilles tendon: a human immunohistochemistry study. Lancet. 2015;385(Suppl 1):19.

9. Park HC, Kim SG, Oh JS, You JS, Kim JS, Lim SC, et al. Early Bone Formation at a Femur Defect Using CGF and PRF Grafts in Adult Dogs: A Comparative Study. Implant Dent. 2016;25:387-93.

10. Qiao J, An N, Ouyang X. Quantification of growth factors in different platelet concentrates. Platelets. 2017;28:774-8.

11. Hu Y, Jiang Y, Wang M, Tian W, Wang H. Concentrated Growth Factor Enhanced Fat Graft Survival: A Comparative Study. Dermatol Surg. 2018;44:976-84.

12. Bozkurt DS, Ongoz DF, Balli U, Atalay EN, Durmuslar MC. Concentrated growth factor in the treatment of adjacent multiple gingival recessions: a split-mouth randomized clinical trial. J Clin Periodontol. 
2015;42:868-75.

13. Takeda Y, Katsutoshi K, Matsuzaka K, Inoue T. The Effect of Concentrated Growth Factor on Rat Bone Marrow Cells In Vitro and on Calvarial Bone Healing In Vivo. Int J Oral Maxillofac Implants. 2015;30:1187-96.

14. Chen X, Wang J, Yu L, Zhou J, Zheng D, Zhang B. Effect of Concentrated Growth Factor (CGF) on the Promotion of Osteogenesis in Bone Marrow Stromal Cells (BMSC) in vivo. Sci Rep. 2018;8:5876.

15. Kim JM, Sohn DS, Bae MS, Moon JW, Lee JH, Park IS. Flapless transcrestal sinus augmentation using hydrodynamic piezoelectric internal sinus elevation with autologous concentrated growth factors alone. Implant Dent. 2014;23:168-74.

16. Kim TH, Kim SH, Sandor GK, Kim YD. Comparison of platelet-rich plasma (PRP), platelet-rich fibrin (PRF), and concentrated growth factor (CGF) in rabbit-skull defect healing. Arch Oral Biol. 2014;59:550-8.

17. Sohn DS, Heo JU, Kwak DH, Kim DE, Kim JM, Moon JW, et al. Bone regeneration in the maxillary sinus using an autologous fibrin-rich block with concentrated growth factors alone. Implant Dent. 2011;20:389-95.

18. Xu F, Qiao L, Zhao Y, Chen W, Hong S, Pan J, et al. The potential application of concentrated growth factor in pulp regeneration: an in vitro and in vivo study. Stem Cell Res Ther. 2019;10:134.

19. Chen X, Chen Y, Hou Y, Song P, Zhou M, Nie M, et al. Modulation of proliferation and differentiation of gingivaderived mesenchymal stem cells by concentrated growth factors: Potential implications in tissue engineering for dental regeneration and repair. Int J Mol Med. 2019;44:37-46.

20. Xu C, Wei Z, Gao H, Bai Y, Liu H, Yang H, et al. Bioinspired Mechano-Sensitive Macroporous Ceramic Sponge for Logical Drug and Cell Delivery. Adv Sci (Weinh). 2017;4:1600410.

21. Xu C, Bai Y, Yang S, Yang H, Stout DA, Tran P, et al. A versatile fabrication strategy of threedimensional foams for soft and hard tissue engineering. Biomed Mater. 2017.

22. Wei F, Li Z, Crawford R, Xiao Y, Zhou Y. Immunoregulatory role of exosomes derived from differentiating mesenchymal stromal cells on inflammation and osteogenesis. J Tissue Eng Regen Med. 2019;13:1978-91.

23. Broege A, Pham L, Jensen ED, Emery A, Huang TH, Stemig M, et al. Bone morphogenetic proteins signal via SMAD and mitogen-activated protein (MAP) kinase pathways at distinct times during osteoclastogenesis. J Biol Chem. 2013;288:37230-40.

24. Wu X, Chim SM, Kuek V, Lim BS, Chow ST, Zhao J, et al. HtrA1 is upregulated during RANKL-induced osteoclastogenesis, and negatively regulates osteoblast differentiation and BMP2-induced Smad1/5/8, ERK and p38 phosphorylation. Febs Lett. 2014;588:143-50.

25. Caddy JC, Luoma LM, Berry FB. FOXC1 negatively regulates BMP-SMAD activity and Id1 expression during osteoblast differentiation. J Cell Biochem. 2020;121:3266-77.

26. Caddy JC, Luoma LM, Berry FB. FOXC1 negatively regulates BMP-SMAD activity and Id1 expression during osteoblast differentiation. J Cell Biochem. 2020;121:3266-77. 
27. Rodella LF, Favero G, Boninsegna R, Buffoli B, Labanca M, Scari G, et al. Growth factors, CD34 positive cells, and fibrin network analysis in concentrated growth factors fraction. Microsc Res Tech. 2011;74:772-7.

28. Rodella LF, Favero G, Boninsegna R, Buffoli B, Labanca M, Scari G, et al. Growth factors, CD34 positive cells, and fibrin network analysis in concentrated growth factors fraction. Microsc Res Tech. 2011;74:772-7.

29. Hong S, Li L, Cai W, Jiang B. The potential application of concentrated growth factor in regenerative endodontics. Int Endod J. 2019;52:646-55.

30. Shang Q, Yu X, Ren H, Shen G, Zhao W, Zhang Z, et al. Effect of Plastrum Testudinis Extracts on the Proliferation and Osteogenic Differentiation of rBMSCs by Regulating p38 MAPK-Related Genes. Evid Based Complement Alternat Med. 2019;2019:6815620.

31. Huang J, Yin H, Rao SS, Xie PL, Cao X, Rao T, et al. Harmine enhances type H vessel formation and prevents bone loss in ovariectomized mice. Theranostics. 2018;8:2435-46.

32. Wang L, Wan M, Li Z, Zhong N, Liang D, Ge L. A comparative study of the effects of concentrated growth factors in two different forms on osteogenesis in vitro. Mol Med Rep. 2019;20:1039-48.

33. Zhang $\mathrm{L}, \mathrm{Ai} \mathrm{H}$. Concentrated growth factor promotes proliferation, osteogenic differentiation, and angiogenic potential of rabbit periosteum-derived cells in vitro. J Orthop Surg Res. 2019;14:146.

34. Topkara A, Ozkan A, Ozcan RH, Oksuz M, Akbulut M. Effect of Concentrated Growth Factor on Survival of Diced Cartilage Graft. Aesthet Surg J. 2016;36:1176-87.

35. Jin R, Song G, Chai J, Gou X, Yuan G, Chen Z. Effects of concentrated growth factor on proliferation, migration, and differentiation of human dental pulp stem cells in vitro. $J$ Tissue Eng. 2018;9:1544420383.

36. Masuki H, Okudera T, Watanebe T, Suzuki M, Nishiyama K, Okudera H, et al. Growth factor and proinflammatory cytokine contents in platelet-rich plasma (PRP), plasma rich in growth factors (PRGF), advanced platelet-rich fibrin (A-PRF), and concentrated growth factors (CGF). Int J Implant Dent. 2016;2:19.

37. Honda H, Tamai N, Naka N, Yoshikawa H, Myoui A. Bone tissue engineering with bone marrowderived stromal cells integrated with concentrated growth factor in Rattus norvegicus calvaria defect model. J Artif Organs. 2013;16:305-15.

38. Honda H, Tamai N, Naka N, Yoshikawa H, Myoui A. Bone tissue engineering with bone marrowderived stromal cells integrated with concentrated growth factor in Rattus norvegicus calvaria defect model. J Artif Organs. 2013;16:305-15.

39. Dohan DM, Choukroun J, Diss A, Dohan SL, Dohan AJ, Mouhyi J, et al. Platelet-rich fibrin (PRF): a second-generation platelet concentrate. Part III: leucocyte activation: a new feature for platelet concentrates? Oral Surg Oral Med Oral Pathol Oral Radiol Endod. 2006;101:e51-5.

40. Liu Y, Kalen A, Risto O, Wahlstrom O. Fibroblast proliferation due to exposure to a platelet concentrate in vitro is pH dependent. Wound Repair Regen. 2002;10:336-40. 
41. Mornet E, Stura E, Lia-Baldini AS, Stigbrand T, Menez A, Le Du MH. Structural evidence for a functional role of human tissue nonspecific alkaline phosphatase in bone mineralization. J Biol Chem. 2001;276:31171-8.

42. Douglas TE, Messersmith PB, Chasan S, Mikos AG, de Mulder EL, Dickson G, et al. Enzymatic mineralization of hydrogels for bone tissue engineering by incorporation of alkaline phosphatase. Macromol Biosci. 2012;12:1077-89.

43. Douglas TE, Wlodarczyk M, Pamula E, Declercq HA, de Mulder EL, Bucko MM, et al. Enzymatic mineralization of gellan gum hydrogel for bone tissue-engineering applications and its enhancement by polydopamine. J Tissue Eng Regen Med. 2014;8:906-18.

44. Rawadi G, Vayssiere B, Dunn F, Baron R, Roman-Roman S. BMP-2 controls alkaline phosphatase expression and osteoblast mineralization by a Wnt autocrine loop. J Bone Miner Res. 2003;18:184253.

45. Hashimi SM. Exogenous noggin binds the BMP-2 receptor and induces alkaline phosphatase activity in osteoblasts. J Cell Biochem. 2019;120:13237-42.

46. Wan M, Cao X. BMP signaling in skeletal development. Biochem Biophys Res Commun. 2005;328:651-7.

47. Shi Y, Massague J. Mechanisms of TGF-beta signaling from cell membrane to the nucleus. Cell. 2003;113:685-700.

48. Wang S, Hu S, Wang J, Liu Y, Zhao R, Tong M, et al. Conditioned medium from bone marrow-derived mesenchymal stem cells inhibits vascular calcification through blockade of the BMP2-Smad1/5/8 signaling pathway. Stem Cell Res Ther. 2018;9:160.

49. Zwijsen A, Verschueren $K$, Huylebroeck D. New intracellular components of bone morphogenetic protein/Smad signaling cascades. Febs Lett. 2003;546:133-9.

50. Taylor IW, Wrana JL. SnapShot: The TGFbeta pathway interactome. Cell. 2008;133:371-8.

\section{Figures}


a

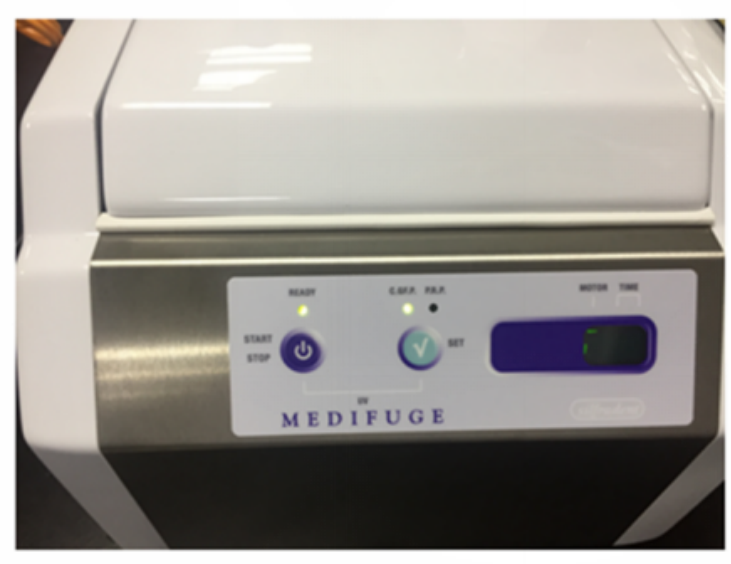

d

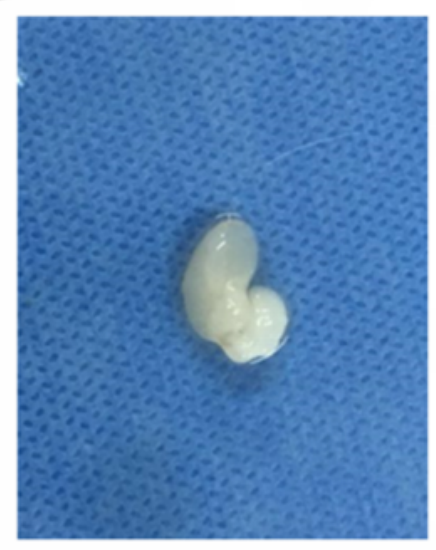

b

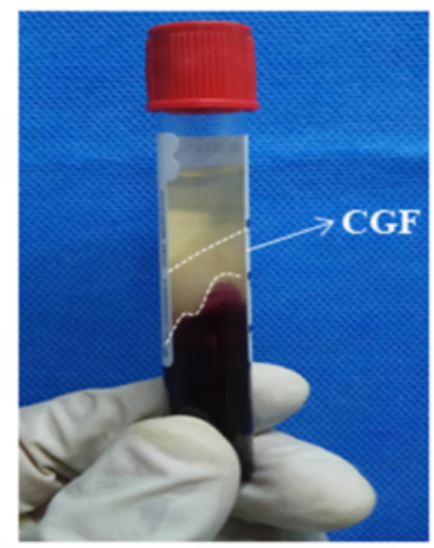

c

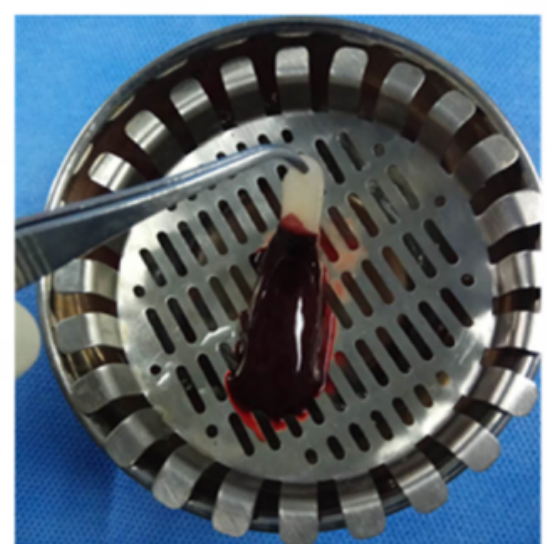

f

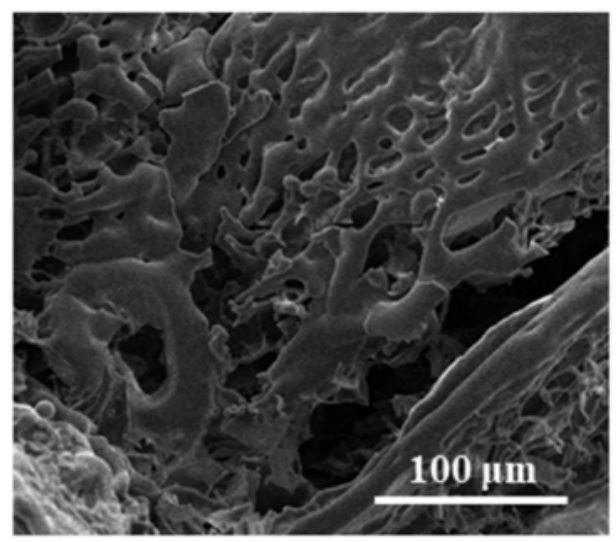

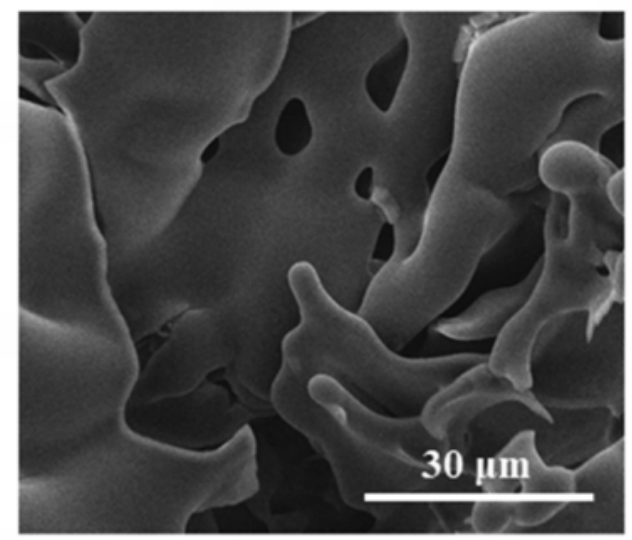

\section{Figure 1}

CGF fabricating and its structure. a Blood from auxiliary veins of SD rats were extracted and centrifuged using Silfradent system. b The blood in the tube after centrifugation. The middle layer indicates CGF (white arrow). c, d CGF gel. e SEM results of CGF in low magnification (Scale bar: $20 \mu \mathrm{m}$ ), a lot of fibrous proteins are found in the figure. $f$ SEM results of CGF in high magnification (Scale bar: $50 \mu \mathrm{m}$ ), the CGF gel prepared by freeze-drying method is porous. 


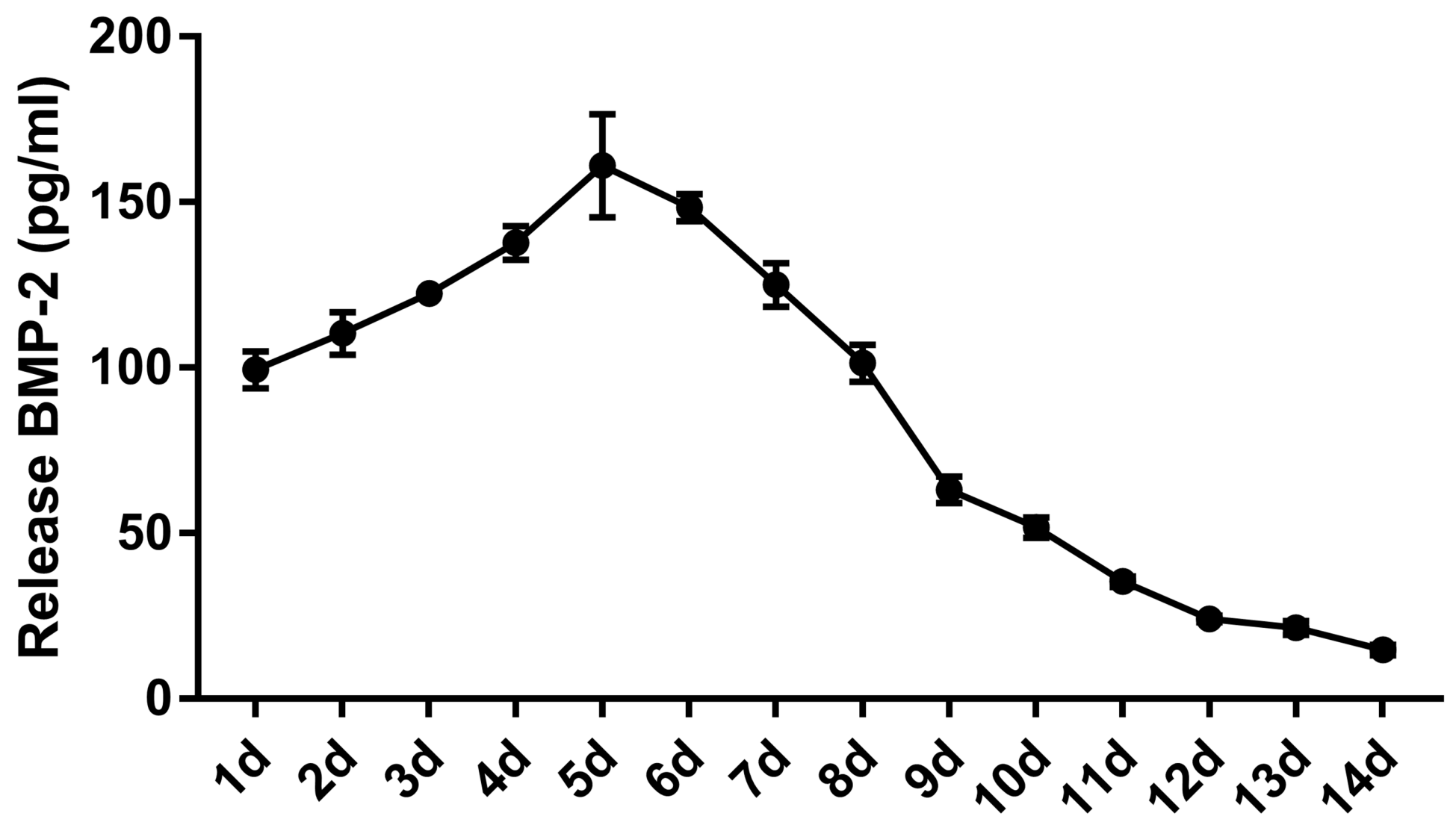

Figure 2

A sustained-release test showed that CGF continuously released BMP-2 for up to 14 days. The level of BMP-2 increased from Day 1 to Day 5. After Day 5, the level slowly decreased to Day 14 . 
a

P0

P1

P3

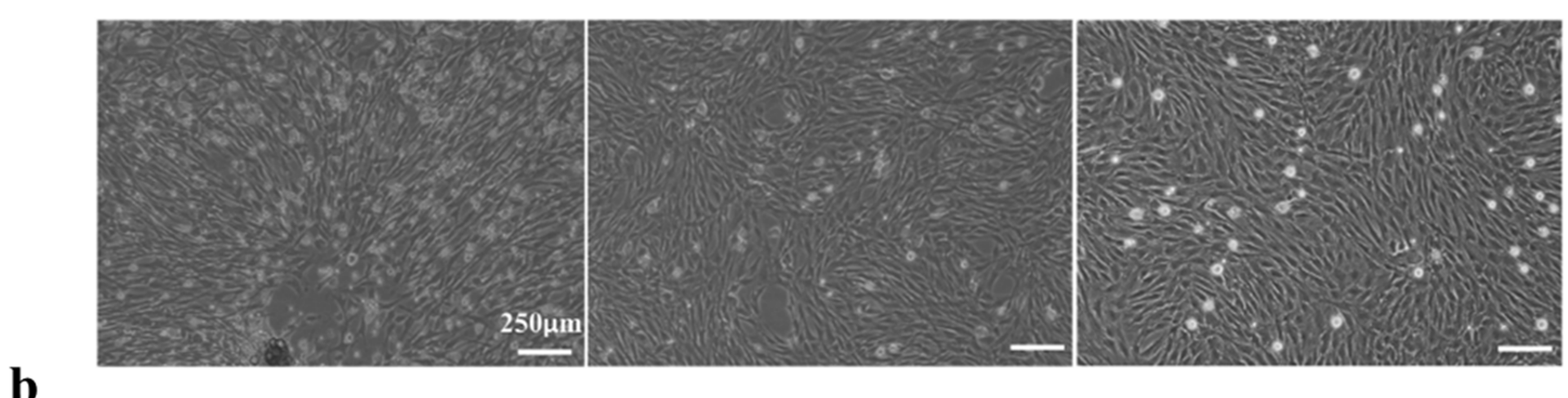

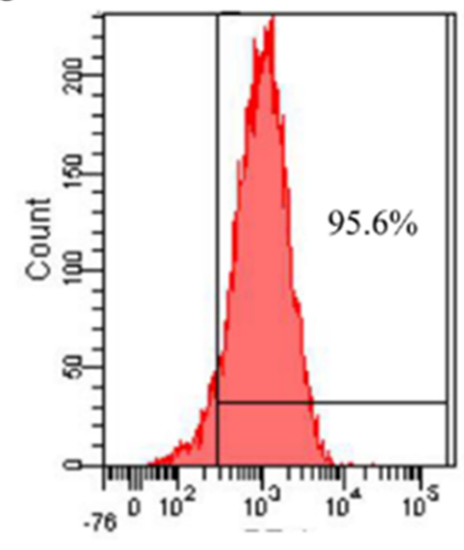

CD-29 FITC

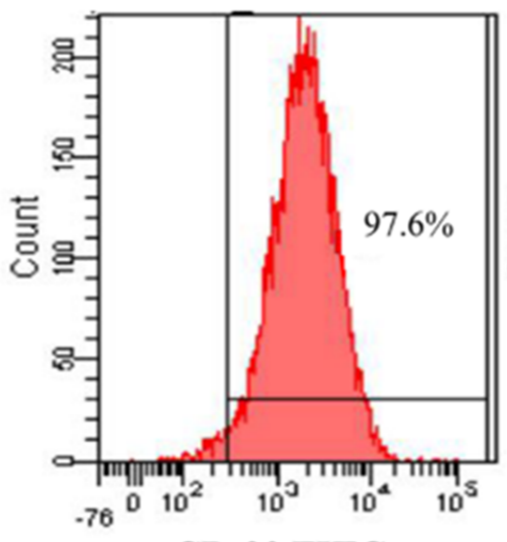

CD-90 FITC

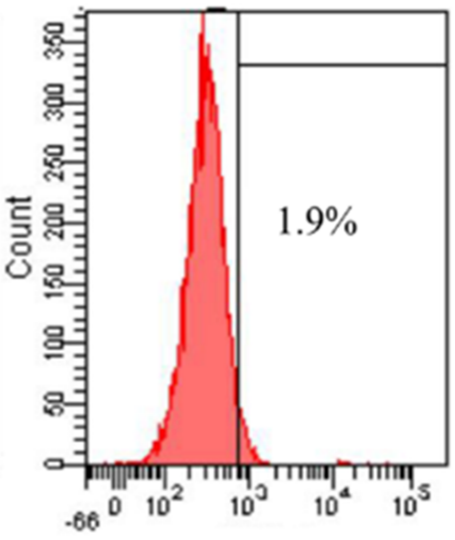

CD-45 FITC

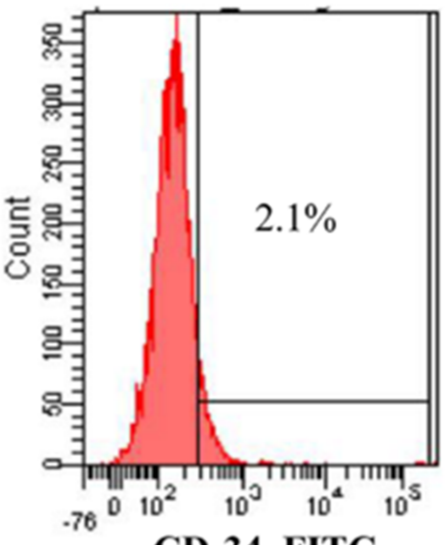

CD-34 FITC

Figure 3

Culture and identification of BMSCs. a BMSCs bone marrow-derived mesenchymal stem cells, P0 passage, $\mathrm{P} 1$ passage, $\mathrm{P} 3$ passage. P0 BMSCs showed short bar shapes, and P1, P3 BMSCs adopted a uniformly spindle-shaped population. b Flow cytometry revealed that BMSCs expressed the surface markers CD29 and CD90, but not CD45 and CD34. Scale bar: $250 \mu \mathrm{m}$. 


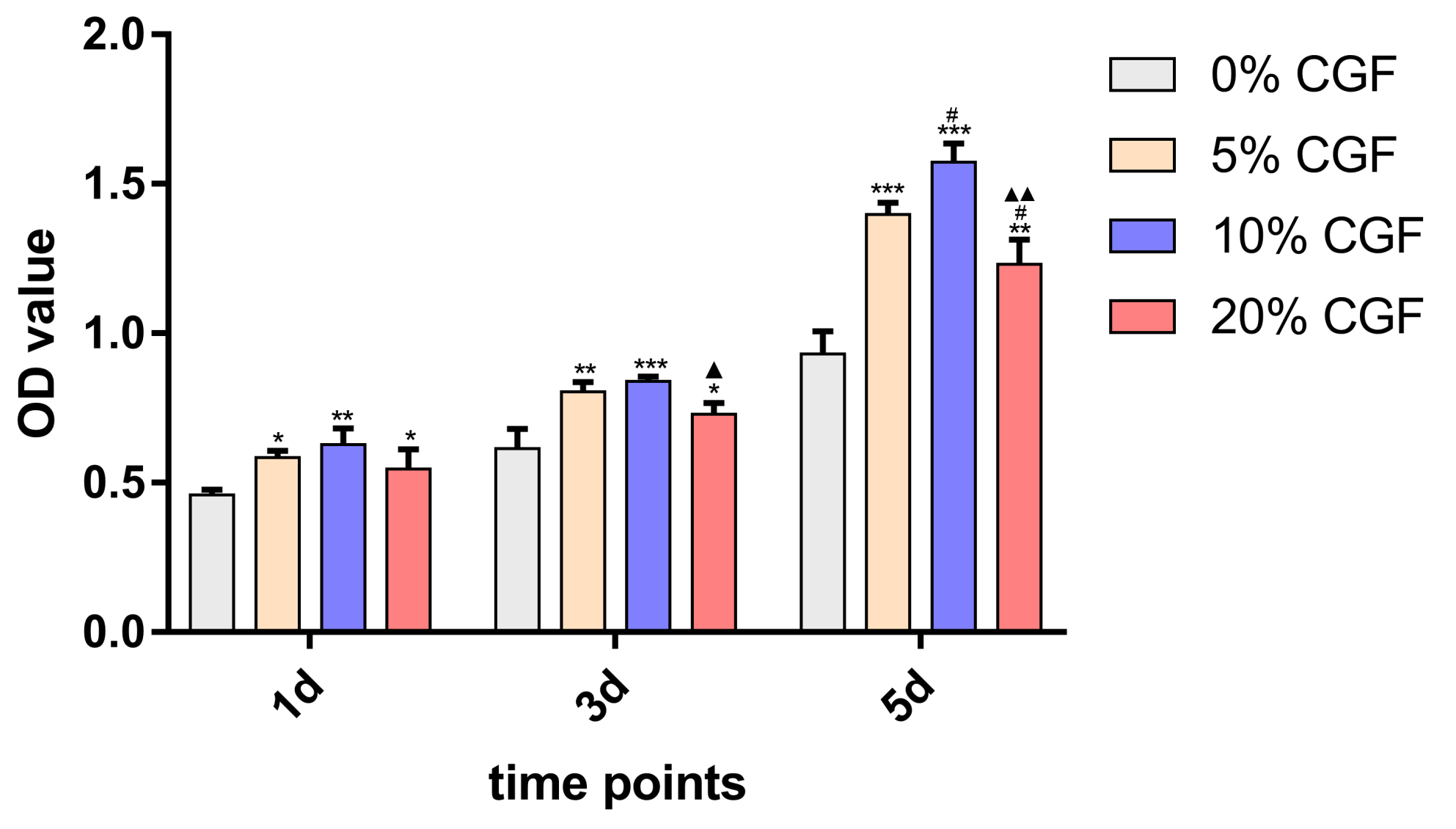

Figure 4

CGF promotes BMSCs proliferation at different time points (1, 3, $5 \mathrm{~d})$. Note: Compared with control group

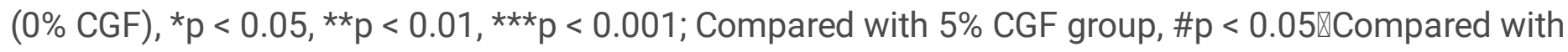
$10 \%$ CGF group, $\Delta p<0.05, \Delta \Delta p<0.01$.

$\mathbf{a}$
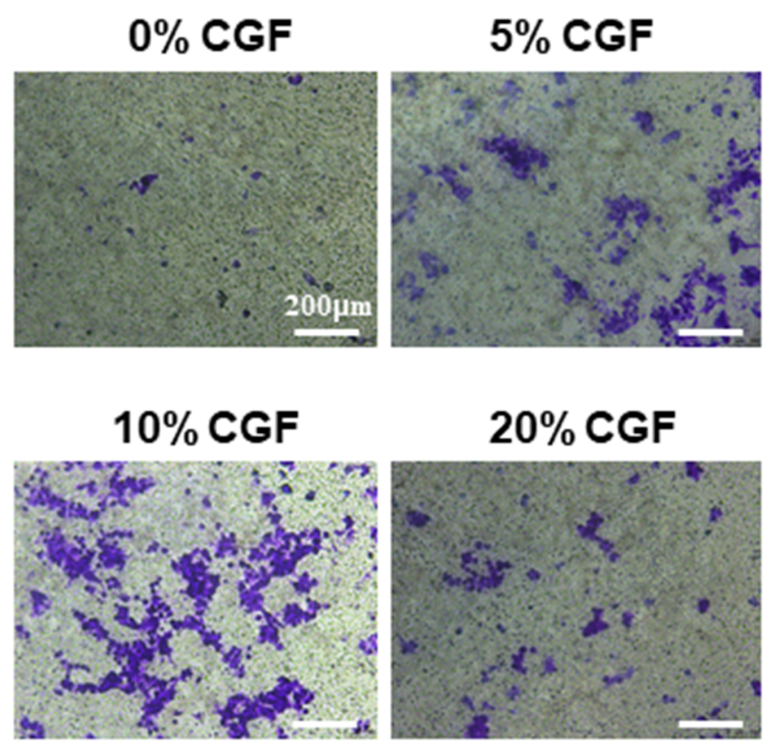

$20 \%$ CGF

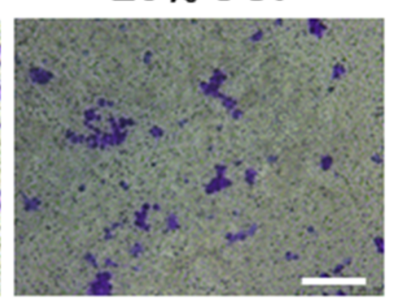

b

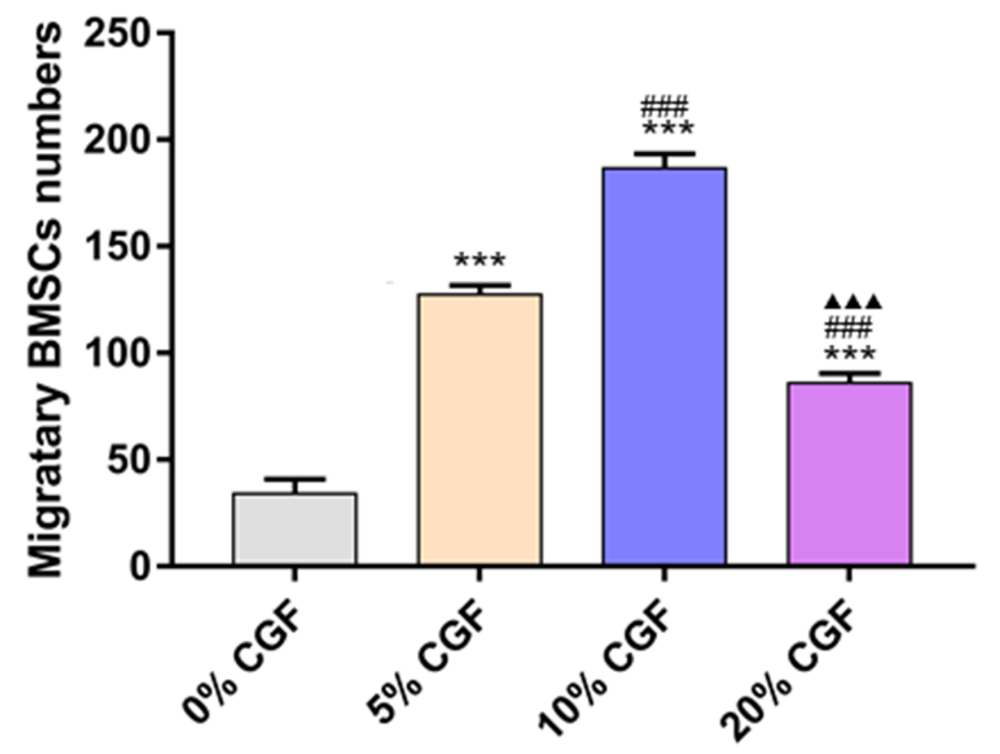

Figure 5 
CGF promotes BMSCs migration. a The crystal violet staining of the migratory cells from different groups after culture in CGF for $24 \mathrm{~h}$. b Representative images were taken using a microscope. (B) Statistical analysis of the average migratory cell numbers per field from different groups at $24 \mathrm{~h}$. Note: Compared with 0\% CGF group (control group); ${ }^{* \star} p<0.001$; compared with 5\% CGF group; $\boldsymbol{\Delta} \mathbf{\Delta} \mathbf{\Delta} p<0.001$; compared with 5\% CGF group.

a

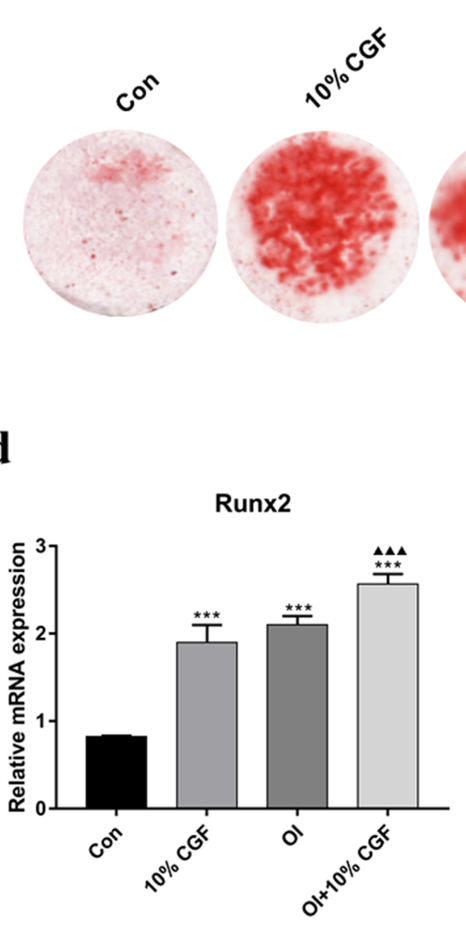

h

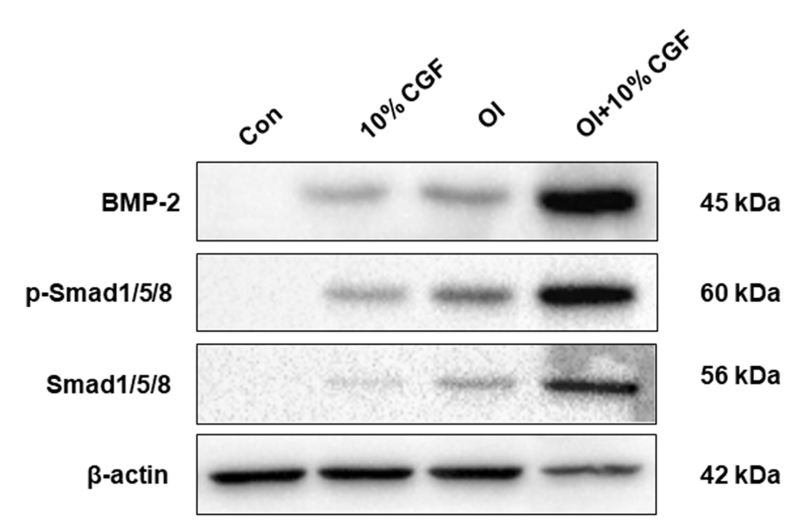

OCN b

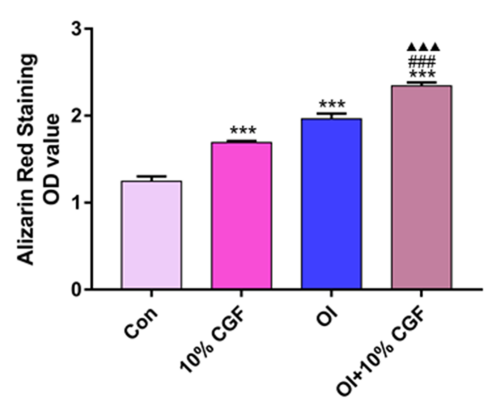

f
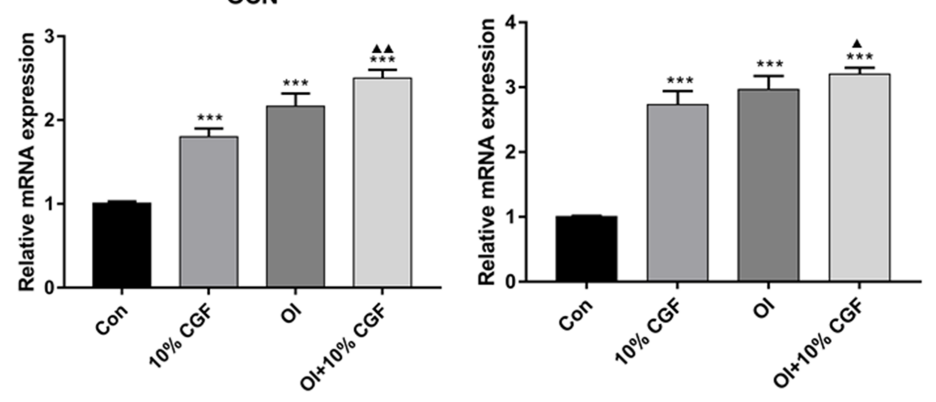

i

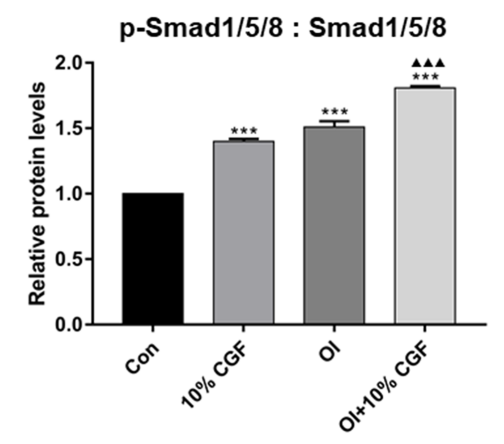

c

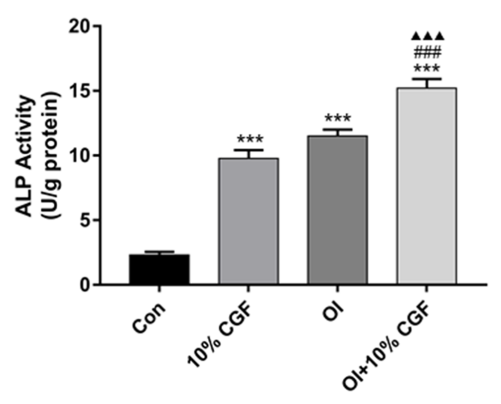

g

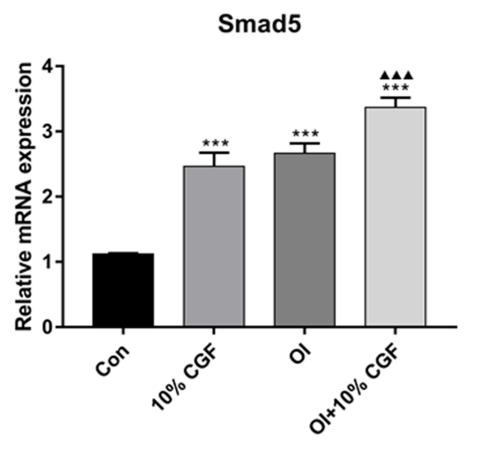

j

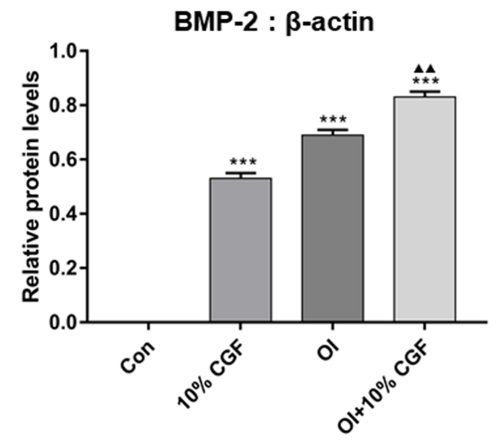

\section{Figure 6}

CGF promotes BMSCs osteoblastic differentiation and osteogenic mineralization via upregulating BMP2/Smad signaling pathway. a The Alizarin Red staining results. b Quantitative analysis of osteogenic mineralization. c ALP activity. The mRNA expression of Runx2 (d), Ocn (e), Smad1 (f), Smad5 (g) detected by RT-qPCR. $\mathrm{h}$ The protein expression of BMP-2, p-Smad1/5/8, and Smad1/5/8 determined by Western blot. $i$ The protein expression ratio of $p-S m a d 1 / 5 / 8$ to Smad1/5/8. j The protein expression ratio of BMP-2 to $\beta$-actin. Note: Compared with control group (OI+0\% CGF), $* \star \star p<0.001$; compared with OI group, $\mathbf{\Delta} p<0.05, \mathbf{\Delta} \mathbf{\Delta} p<0.01, \mathbf{\Delta} \mathbf{\Delta} \mathbf{\Delta} p<0.001$. 


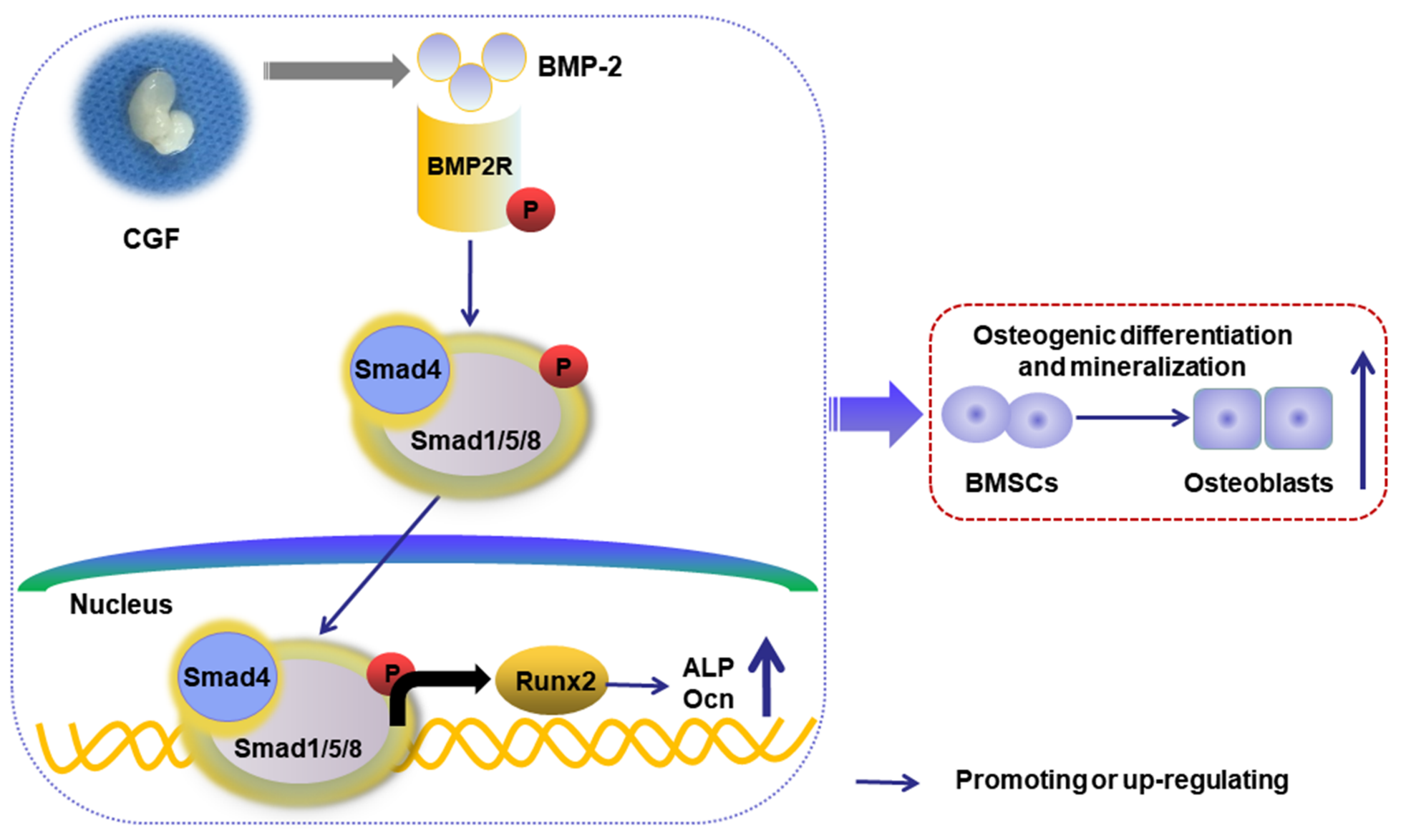

Figure 7

The possible mechanism of CGF promoting osteogenic differentiation and mineralization of BMSCs. The possible mechanism might be that, CGF secretes BMP-2, then the secreted BMP-2 binds to BMP-2R and activates the downstream osteogenic genes through a Smad4 complex, then promotes the expression of phosphorylated Smad1/5/8 and its transfer from the cytoplasm to the nucleus to promote the transcription of Runx2 and subsequent activation of osteogenic genes such as ALP and OCN, finally improve the osteogenic differentiation and mineralization of BMSCs. 